\title{
Explaining the determinants of Human Papillomavirus Vaccine uptake and Strategies to Increase Its Uptake among young adults in Capital of Iran: a qualitative study
}

\section{Soudabeh Yarmohammadi}

school health and saefty,shahid beheshti univversity of medical sciences

Mohtasham Ghaffari

Shaheed Beheshti University of Medical Sciences

Ali Ramezankhani ( $\square$ aramezankhani2018@gmail.com )

school of public health and safety,shahid beheshti university of medical sciences https://orcid.org/0000-0002-3109-1641

\section{Research article}

Keywords: Human Papillomavirus Vaccine, Young Adult, Strategie, Qualitative Study

Posted Date: March 30th, 2020

DOI: https://doi.org/10.21203/rs.3.rs-19841/v1

License: (c) (1) This work is licensed under a Creative Commons Attribution 4.0 International License.

Read Full License 


\section{Abstract}

Background The HPV vaccine prevents the most common cancer in women, which is cervical cancer. This study aimed to determine the factors affecting the uptake and non- uptake of HPV vaccine in the young adult in Tehran and strategies to increase their vaccine uptake. Methods This is the first qualitative study with the conventional content analysis approach in this context in Iran in 2018-2019. Participants, with a maximum variation in terms of age, sex, educational level, occupation, income, work experience, marital status, were selected purposefully to take part in a semi-structured interview. The data were recorded and analyzed according to the interview continued until data saturation. MAXQDA software version 10 was used for data management. Results The participants were 20 health professionals and 10 young adults from Tehran. Factors affecting HPV vaccine uptake and non- uptake identified as seven categories, including knowledge, health value, external stimuli, outcome expectations, perceived threat, healthcare services, and Contextual factors. Moreover, the strategies to increase vaccine uptake included Compulsory vaccination, vaccine availability, and implementing the educational process. Conclusions Considering the factors identified in this study, the authorities and practitioners must take steps to increase the HPV vaccine in young adults by considering the effective factors, as well as the strategies, such as Compulsory vaccination, increasing vaccine availability, and implementing educational interventions.

\section{Background}

Cancer of the cervix was the fourth most common cancer in women worldwide in 2012, with the majority of cases observed in developing countries [1]. A significant risk factor for the extension of cervical cancer is long-term infection with high-risk strains of human papillomavirus (HPV) [2]. HPV 16 and 18 are the most common types identified in women and estimate for $70 \%$ of all cervical cancer. Less it is currently understood about male HPV-attributable cancers. Thirty- HPV-associated cancers eight percent diagnosed in men, with oropharyngeal being the most common [3]. Estimations of prevalence indicate that about 79 million individuals infected with HPV, and approximately 14 million people become infected each year [4].

The human papillomavirus (HPV) vaccine can as a primary prevention tool [5]; vaccination reduces HPVassociated diseases like cervical cancer, vulvar cancer, anal cancer, and genital warts [6]. The vaccine first became widely available in 2006 for females [7], And 2010 for males [8], and vaccination rates have steadily increased since then $[9,10]$. Currently, the HPV vaccination among young adults $(19-26$ years of age) with at least one dose ever is $8 \%$ for men $40 \%$ for women [11]. And Mortensen et al. identified two major factors influencing vaccination uptake - knowledge and structural incentives: 'When structural barriers are low, as in Italy and the UK, acceptability is high, but knowledge does not necessarily follow. When structural barriers are higher, as in Germany and France, the need for knowledge and reassurance is higher [12]. 
Studies indicate that the following factors were most likely to be associated with future acceptance of the HPV vaccine perceived risks of the vaccine, perceived effectiveness of the vaccine, perceived barriers (e.g., vaccine costs, concerns that the vaccine would promote adolescent sexual behavior), physicians' recommendations and the opinions of significant others (i.e., subjective norms) [13-16]

Human Papilloma Virus (HPV) knowledge is an essential determinant for vaccine acceptance and uptake $[17,18]$. Very many studies have measured knowledge or awareness of HPV/HPV vaccination $[19,20]$. An international review of surveys of young women aged up to 26 years reported poor knowledge and misconceptions about HPV and its vaccine [21].

Moreover, a meta-analysis of studies centering on European adolescents reported a poor perception of basic HPV and HPV vaccine knowledge [22].

Few people in Iran use the HPV vaccine, which is one of the most important causes of the lack of knowledge among the public about the vaccine in Iran and when it is injected [23].

Because the HPV vaccine has only recently introduced worldwide, most studies have focused on determinants of future acceptance of the HPV vaccination. None of the studies investigated the causes of uptake and non-uptake between boys and girls. The purpose of this study was to determine the causes of vaccine uptake and non-uptake among girls and boys aged 18 to 27 years and to determine strategies for increasing vaccine uptake in a low vaccine country.

\section{Methods}

\section{Study design:}

An exploratory qualitative study was conducted using in-person and face-to-face interviews with a health professional and young adult.

\section{The participants and the study setting}

The present study included 30 participants, including 20 health professionals in various fields (health education and health promotion, gynecology, infectious diseases, dermatology, urology, reproductive health, social practitioner, psychologist) and ten young adults in the age range of 18-27 by purposeful sampling with maximum variation in age, sex, educational level, occupation, income, work experience, marital status. The health professional inclusion criteria included having experience and knowledge about HPV and informed consent to participate in the study. The inclusion criteria for young adults included knowing HPV, informed consent to participate in the study, and the age range of 18-27. The research environment was hospitals affiliated to Shahid Beheshti University of Medical Sciences.

\section{Data collection}


The authorization was received from Shahid Beheshti University of Medical Sciences for 12 hospitals. By referring to different hospitals and talking to the health professional, they were asked to participate in the interview. Young participants were also asked to be interviewed, either as a patient or companion, and if accepted, they offered the time and place of the interview. The interviews were conducted in a place where was most appropriate for the participants (for example, in the hospital and at the university). No one was present at the interview except the researcher and the participants. Each interview began with the purpose of the study, and the participants voluntarily participated in the study. They were asked to answer demographic questions, and then the interview began. The interview guide is listed below. The interviews were recorded, and each interview generally lasted 30 to $60 \mathrm{~min}$. Data collection continued until further information was obtained, and the study saturated with 29 interviews. The interviews were conducted from May to November in 2019, and each interview was transcribed immediately after that. All interviews were conducted by the main researcher (S.Y).

\section{Interview Guide}

A semi-structured interview guide was used. The main question asked from the health professionals was: "What do you think of the barriers and facilitators of uptake and non- uptake of HPV vaccine by young adults?"

The main question asked from the young adults was: "Which factors encourage or prohibit you from uptake HPV vaccine?". Follow-up and probe questions were also used, such as: "Can you explain more about it?". Two pilot interviews were conducted before the study began. These interviews were not included in the present study. After pilot interviews, there was no significant change in the interview guide.

\section{Data analysis}

Data analysis was performed simultaneously with data collection using Graneheim and Lundman's content analysis approach [24]. This method can reveal the hidden themes and patterns of the study from the content of the data obtained from the participants [25].

Immediately after the interview, the interview was transcribed. The transcripts were read several times to get an overall picture of what was said. The transcripts of the interviews were then entered into the MAXQDA Version 10 software. Subsequently, semantic units consisting of related sentences or paragraphs were identified, and the initial codes were extracted. The codes were merged and categorized according to similarities and differences and named as subcategories at different abstracts levels [19]. Regarding the controversial codes, the researchers argued and agreed.

To establish reliability in this study, the four strategies recommended by Schwandt et al. were used [26], including credibility, confirmability, transferability, dependability. To establish credibility, continuous and prolonged engagement researcher involvement used in the present study. Peer check was conducted through the research team by holding meetings and discussing the data and analysis among the researchers themselves and with the specialists. Furthermore, all interviews were recorded and 
transcribed into written versions. The interviews were summarized, and the data and codes were analyzed by the member check and their feedback was considered. Confirmability indicates the relationship between the data and the resources used. The observance of this criterion emphasizes that the results of the study are not relevant to the researcher's knowledge [27]. Data Confirmability was performed by the main researcher by reviewing and collecting the ideas of other researchers and obtaining relevant studies documentation. Moreover, the Confirmability or review method was performed by the supervisors. To this end, the interviews, codes, and classes obtained by several experts were studied.

Transferability in this study was performed by providing a comprehensive description of the topic, participants, method of data collection, and analysis. To achieve dependability, detailed, and accurate note-taking was also used to provide details to other researchers to study or develop similar cases.

\section{Ethical considerations}

The Ethics Committee approved the study at a meeting of the Faculty of Health and Safety at the Neuroscience Research Center of Shahid Beheshti University of Medical Sciences. Moreover, some cases were observed, including obtaining consent from the participants, anonymity, confidentiality, obtaining permission to record audio interviews, and the right to withdraw when they desired.

\section{Results}

In this study, data saturation was obtained after 30 interviews. Finally, the data were categorized into 20 subcategories and seven categories. A total of 20 health professionals in different specialties and nine young adults participated in the study. Their mean age was $2.40 \pm 0.224$ and $24.86 \pm 3.761$, respectively. The participants' specifications are presented in Tables 1 and 2.

The Qualitative content analysis led to seven categories, including health value, knowledge, external stimuli, outcome expectations, perceived threat, healthcare services, and Contextual factors (Table 3).

\section{Health value}

This category consists of two subcategories, including taking care of health and taking responsibility for health.

Females uptake the vaccine to maintain their health and that of their partners, while males do not care about their health and uptake the vaccine only to maintain their partner's health.

"Girls uptake the vaccine for their health as well as those around them" (Young participant,27 years old).

The participants believed that males do not usually refer to a physician for their illness, and they believe the disease was unrelated to them.

"The problem of men in our society is that they care too much about themselves; they put all burden on women's shoulders. Go and uptake, and like preventing pregnancy from closing the tube, most men do 
not put the burden on their shoulders." (Young participant, 27 years old)

\section{Knowledge}

This category comprises two subcategories, including young people's lack of knowledge about HPV and its vaccine and its benefits, and lack of knowledge about health service clinics and the place to purchase the vaccine. Most of the participants emphasized the lack of knowledge about the disease, the vaccine, and its associated benefits.

"I did not inject the vaccine because I do not even know whether or not there is a vaccine, I do not even know its name" (Young participant, 27 years old)

"I google the address of pharmacies that had the vaccine in cities and Tehran for my students and told them. How should people know? Where should they buy the vaccine?" (Gynecologist, 63 years old)

\section{Outcome expectations}

This category consists of two subcategories, including the fear of vaccine side effects, the uncertainty of the vaccine's outcome.

Side effects, such as infertility of girls by vaccine uptake, can be a cause for both young people and parents as well as some physicians not to uptake the vaccine.

"If in the future, my daughter gets an ovulation disorder by uptake the vaccine, what should I do if something wrong will happen? It is my mistake that I decided for her." (Reproductive Health Specialist, 40 years old)

The participants were not sure of the benefits of the vaccine and feared that they would become ill with the vaccine.

"People's trust in the medical system has diminished, and this can play a role in non-uptake. She says maybe I will become ill with the vaccine" (psychologist, 31 years old)

\section{Perceived threat}

This category consists of two subcategories called perceived danger, perceived severity.

The perceived danger is the belief that the young person is likely to get the disease. Most of the participants believed that girls were more at risk of illness than boys. Moreover, a lack of confidence in a future partner and having multiple partners are factors for vaccine uptake. Some of the participants did not consider themselves susceptible to the disease due to a lack of sex.

The boys believed that they are not vulnerable to the disease; since they have the devices to prevent sexually transmitted diseases, and they could prevent the disease. The factor that prompts the boys to 
uptake the vaccine is that their genitalia becomes ugly due to the disease, and as a result, their partner will avoid them.

"The boys in my relatives are not impressed. I talk to them about these issues, but it is useless. Unless I show them a picture of the disease and tell them you may be like this in the future, for example." (Healthy young participant, 25 years old)

"Using condoms can make men think they are not vulnerable, and will never have a problem" (Psychologist, 31 years old)

The perceived severity is the belief that young people have about the losses from no-vaccination. Most of the participants reported that the cause of vaccination in girls is the fear of cancer.

"The most important cause of the uptake is fear, fear of cancer. This fear increases anxiety and causes to inject this vaccine." (Psychosexualist, 45 years old)

\section{Contextual factors}

This category consists of four subcategories, including education role, sex role, personality characteristics, and culture effects.

The participants mentioned that educated people find it easier to accept the health professionals' words.

"Education is important for both knowledge and acceptance of the health professionals' words and regular visits to the physician and various health professionals. Furthermore, it can be effective in vaccine uptake." (Young participant, 23 years old)

Women are usually more likely to uptake vaccines because of their gender role of being a mother. Men, on the other hand, think that they do not need the vaccine because they are empowered. Men also consider the HPV vaccine a women-only vaccine and believe it has nothing to do with them.

"Boys think they do not get the disease at all because they think men are strong enough not to get it" (Reproductive Health Specialist, 49 years old)

Risky individuals are more likely to uptake vaccines than others, and some do not uptake the vaccine due to the fear of the needle.

"After finding out about the vaccine, I did not uptake again because I was afraid of the needle." (Patient, 17 years old)

Culture is also one of the most effective factors for the vaccine uptake. The participants mentioned parents' failure to talk to their children about sex issues because of the values and culture prevalent in the society, as well as the fear of others' negative view about them by vaccinating. 
"The culture and values of the society is very important to a woman. And because of this fact she may not uptake the vaccine." (Health Education Specialist, 58 years old)

\section{External stimuli}

This category consists of five subcategories, including peer effects, parental effects, physician effects, media effects, and observational learning.

In peer effects, usually, friends, who have knowledge, information, and experiences about the disease and HPV vaccine, can be useful in uptake the vaccine.

"Peers can be effective if they are aware of the disease." (Reproductive Health Specialist, 40 years old)

Parents believe that uptake the vaccine by their children will increase their sexual freedom. Parents' lack of knowledge about HPV and its vaccine, family religiousness, and lack of belief in sexual intercourse outside marriage in their children can be the reasons for non-vaccination.

"Parents say that if their children get the vaccine, they can go and do any sexual activity." (Health Education and Health Promotion, 36 years old)

Concerning the physicians' effects on vaccine uptake, the participants believed that physicians could be a contributing factor to the vaccine uptake by scaring the patient about the disease risk. They also sometimes deny the illicit sex of young adults because of their religious affiliation.

"When physicians scare the patient about the disease and its complications, then patients search the internet, and they will be terrified. Then they come back and ask for the vaccine uptake." (Gynecologist, 60 years old)

The media has created confusion in public with anti-vaccine advertising and misinformation about the vaccine side effects, and this can be a reason for non-vaccination.

In observational learning, the participants believed that young adults by modeling those who uptake the vaccine and seeing those who were protected with HPV vaccine uptake could be encouraged to uptake the vaccine.

"When a person sees those who uptake and finds that they have not been harmed and have been protected against the disease, it could be an encouragement to uptake the vaccine." (Health Psychologist, 40 years old)

\section{Health services}

This category consists of two subcategories, including costs and service coverage.

Most of the participants mentioned the high cost of the vaccine as a reason for non-vaccination. They also demanded the government free vaccines or covered by insurance. Another factor that has delayed 
vaccine uptake was that young adults were busy. Moreover, the vaccine requires a three-period injection, which creates a barrier to uptake. It creates a barrier to uptake. The participants believed that health care providers' behavior could also affect vaccine uptake and non-uptake. III-treatment of the patient with the patient is a known cause of vaccine failure. Inappropriate physician's behavior with the patient is a known cause of non-uptake.

"The vaccine is costly, and it is a financial issue, and we do not uptake it." (Dermatologist, 64 years old) "One of the barriers to vaccine uptake can be time and being busy." (Reproductive Health Specialist, 55 years old)

The participants believed that one of the reasons for the non-uptake in young adults is the vaccine shortage and not be readily available as well as the distance from the uptake location and the inappropriate place for the vaccine uptake privacy.

"I wanted to buy the vaccine. I even called the biggest pharmacy, I called the clinics, and they had no vaccine." (Young participant, 27 years old)

"Most of my patients who bought the vaccine could not uptake it. I even told some of them to bring it, and I do the uptake for them." (Gynecologist, 60 years old)

"I had patients coming from another doctor's office while they were crying. Even one of the doctors once called the police to take the patient and hand them over." (Reproductive Health Specialist, 49 years old)

\section{Strategies to increase vaccine uptake in a low-vaccine country}

During the interview, all participants were asked to make suggestions on vaccine uptake enhancement strategies, including providing free vaccine, vaccine availability, and the educational process (Table 4).

\section{Discussion}

In this study, factors affecting uptake and non- uptake HPV vaccine were evaluated from the perspective of health professionals and young adults, and also the strategies to increase vaccine uptake were identified.

In the present study, the majority of the participants mentioned the lack of knowledge on HPV and HPV vaccine as an effective factor for non-uptake vaccination. This may be due to a person's unwillingness to be aware of the disease and its vaccine, on the other hand, due to the culture and values of the society where talking about sex is thought taboo. Many studies have also reported that lack of HPV vaccine uptake is due to a lack of knowledge about the vaccine and related cancer in men and women [28-31].

The vaccine cost was reported as a major obstacle in this study. On average, each dose of the vaccine in Iran is 325,000 tomans, meaning that a complete vaccine series is 975,000 tomans. Many people spend more money on trivial things compared to the vaccine cost; since the importance of uptake, the vaccine 
has not yet been determined. Many people did not do the uptake vaccination due to its high cost. Many studies have also mentioned cost as a barrier to HPV vaccine uptake [32-34]. In the present study, girls tended uptake the vaccine due to the lack of trust in their future sexual partner, vulnerability to infectious diseases, fear of cancer, and boys due to fear of the appearance of the disease and transmission of the disease to their sexual partner. Some of the participants stated that some boys were reluctant to uptake vaccines because of the use of condoms to prevent sexually transmitted diseases and their invulnerability to the disease.

In the study of Kwan et al., some participants reported that they did not receive uptake vaccine by the age of 18 and above; since they did not intend to have sex [35]. Moreover, in the study of Marlow et al., they reported that specific religious beliefs about avoiding sexual intercourse outside marriage have caused some women to feel that the risk of HPV will not be worrying [36]. Many studies have also cited fear of cancer and its prevention as a reason for the vaccine uptake [37-41].

The present study showed that friends could be effective in the uptake of the vaccine. The study of Katz et al. showed that adults feel that peers provide inaccurate information about sex to their children and encourage one another to engage in high-risk behaviors. At the same time, adolescents acknowledge the positive impact of peers, especially on encouraging uptake vaccines [32]. The participants in the study by Francis et al. also pointed to the role of friends in decision making for health services [42].

In the present study, factors preventing parents from vaccination of their children included parents' difficulty in talking to their children about sex issues, beliefs about sexual freedom in children with vaccination, and parents' fear of children being infected with the disease by vaccine uptake, and belief in lack of sexual intercourse outside marriage in children. Several studies have also pointed to these factors $[35,37-39,43,44]$.

Physicians also had an encouraging and inhibiting role in the vaccine uptake. Physicians' advice may be more effective than others in the uptake of the vaccine. Sometimes physicians may also have religious bias and deny having the disease and do not consider the vaccine uptake necessity. Several studies have also pointed to these factors $[33,45,46]$.

In the present study, the participants noted the inaccurate advertising about the vaccine in the mass media as well as creating confusion among the public, which may cause non-uptake of the vaccine. The study by Cover et al. showed that the media, with positive and negative messages, may affect people's decision to uptake the vaccine [40].

In the current study, taboo and stigma are reported as the other factors of non-uptake of the vaccine. In most of the areas studied (lack of knowledge about HPV disease and the HPV vaccine, perceived threat, external stimuli), there are traces of taboo and stigma. They included parents' shame about talking about sex with children, fewer men's referrals to the physician due to their shame, lack of vaccination due to the prevailing atmosphere in the society and family, and misbehavior of the staff with people with sexual problems. Several studies have also pointed to these factors [35, 47-49].

Page 10/21 
In the present study, the most important recommendations of young adults and health professionals for increasing vaccine uptake strategies are to educate different people to become familiar with HPV and HPV vaccine. By focusing on the education process, which includes the trainer, the learner, the conditions of education, the content of the education, the timing and channels of knowledge transfer and awareness, the proper training can be implemented.

In the present study, the participants preferably suggested the health professionals as trainers; since they are more reliable and knowledgeable. Moreover, the importance of the role of virtual networks and their impact on vaccine uptake, including mass media, can be the essential source of information for health professionals. Various studies have also emphasized the role of physicians and the media in knowledge transfer $[35,39,43]$.

Given the culture of the society, it is difficult to talk about sex issues, especially sexually transmitted diseases. These issues are better to be educated individually. In the study by Yarmohammadi et al., it was believed to provide sex education individually [50]. Information should also be provided step-by-step from school to the elderly period, and there should be proper planning and compulsory education in sexually transmitted diseases. Health education models should also be used to change behavior in educational content design. In the study by Ferrer et al., most female participants reported that they received the vaccine information through school and classrooms [51].

The learner could be teenagers, youth, parents, and health professionals, educational content is designed for each one according to their knowledge and awareness, as well as the cultures and values of each country. Fontenot's study showed that the family had a facilitating role in vaccine uptake and should be educated about HPV and the vaccine [48].

In the study by Tissot et al., the participants generally agreed that written material should be provided separately for teenagers, youth, and parents. They also suggested that educational materials, including graphic images of HPV, are effective in emphasizing the infection for teenagers and youth [52].

\section{Strengths}

The strength of this study was that some of the health professionals and young adults were mothers and stated the factors of uptake and non-uptake vaccinate from their maternal perspective, which made the study information more complete Given that the study results are also transferable to younger ages, the other strength of the study was that the maximum variable was considered in terms of demographic characteristics between the participants. The study also asked the views of both sexes about the vaccine uptake, and the important strength was that the young adults in the study decided to the vaccine uptake.

\section{Limitations}

This study also had limitations. Qualitative studies are not inherently generalizable; therefore, more attention needs to be paid to the population when using the findings of this study. Also, it was difficult to interview HPV patient participants and those who received the vaccine due to the community culture. 


\section{Conclusion}

In conclusion, our study indicates that deciding about uptake or non-uptake of the HPV vaccine is a multilateral decision for young adults. With associated with knowledge, trust in the vaccine benefit, social norms and values, perceived danger of HPV vaccine and cervical cancer, parental approval, sex partners, friends and doctors, and sexual activity. This study also helps to increase HPV vaccine uptake in Iranian youth. If vaccination coverage has to be improved, there is a pressing need for culture-appropriate education about the HPV vaccine and health care providers' recommendations.

\section{Declarations}

\section{Acknowledgements}

The research team would like to thank the School of Health and Safety of Shahid Beheshti University of Medical Sciences for supporting this study. They also would like to thanks the study of health professionals and young adults.

\section{Authors' contributions}

AR conceptualized and designed the project, and obtained research funding. AR and MGH provided feedback on the Project. SY led the analysis of the transcripts, and with AR developed the manuscript. All authors reviewed and approved the final version. SY and AR are the guarantors of the manuscript. SY is the lead author, and AR is the senior author. Other authors are listed in order of contribution.

\section{Authors' information}

1. PhD Student in Health Education and Promotion, School of Public Health and Safety, Shahid Beheshti University of Medical Sciences, Tehran, Iran. 2. Professor of Health Education and Promotion Department of Environmental and Occupational Hazards Control Research Center, School of Public Health, Shahid Beheshti University of Medical Sciences, Tehran, Iran. 3. Professor, Department of Public Health, School of Health, Shahid Beheshti University of Medical Sciences, Tehran, Iran

\section{Funding}

This study is funded by a research grant from the School of Health and Safety of Shahid Beheshti University of Medical Sciences. The funding body (SBMU) didn't have any role in the design of the study and collection, analysis, and interpretation of data and in writing the manuscript.

\section{Availability of data and materials}

Data is available by the corresponding author request.

\section{Ethics approval and consent to participate}


The Ethics Committee approved the study at a meeting of the Faculty of Health and Safety at the Neuroscience Research Center of Shahid Beheshti University of Medical Sciences. Moreover, some cases were observed, including obtaining consent from the participants, anonymity, confidentiality, obtaining permission to record audio interviews, and the right to withdraw when they desired.

\section{Consent for publication}

Not applicable.

\section{Competing interests}

The authors express that they have no competing interests.

\section{References}

1. Gaffney DK, Suneja G, Ryu SY, McCormick M, Plante M, Mileshkin L, et al. The cervix cancer research network: A global outreach effort on behalf of the gynecologic cancer intergroup. International Journal of Radiation Oncology• Biology• Physics. 2015;92(3):506-8.doi:

10.1016/j.ijrobp.2015.02.054

2. Bosch FX, Lorincz A, Muñoz N, Meijer C, Shah KV. The causal relation between human papillomavirus and cervical cancer. Journal of clinical pathology. 2002;55(4):244-65.

3. Markowitz LE, Dunne EF, Saraiya M, Chesson HW, Curtis CR, Gee J, et al. Human papillomavirus vaccination: recommendations of the Advisory Committee on Immunization Practices (ACIP). Morbidity and Mortality Weekly Report: Recommendations and Reports. 2014;63(5):1-30.

4. Prevention CfDCa. HPV Vaccine-Questions \& Answers. http://wwwcdcgov/vaccines/vpdvac/hpv/vac-faqshtm. 2014.

5. Markowitz LE, Tsu V, Deeks SL, Cubie H, Wang SA, Vicari AS, et al. Human papillomavirus vaccine introduction-the first five years. Vaccine. 2012;30:F139-F48.doi:

https://doi.org/10.1016/j.vaccine.2012.05.039

6. Arbyn M, Xu L, Simoens C, Martin-Hirsch PP. Prophylactic vaccination against human papillomaviruses to prevent cervical cancer and its precursors. Cochrane Database of Systematic Reviews. 2018(5).doi:https://doi.org/10.1002/14651858.CD009069.

7. Control CfD, Prevention. Progress toward implementation of human papillomavirus vaccination-the Americas, 2006-2010. MMWR Morbidity and mortality weekly report. 2011;60(40):1382.

8. Lee HY, Lee J, Henning-Smith C, Choi J. HPV literacy and its link to initiation and completion of HPV vaccine among young adults in Minnesota. Public health. 2017;152:172-8.doi: https://doi.org/10.1016/j.puhe.2017.08.002

9. Stokley S, Jeyarajah J, Yankey D, Cano M, Gee J, Roark J, et al. Human papillomavirus vaccination coverage among adolescents, 2007-2013, and postlicensure vaccine safety monitoring, 2006-2014 -United States. MMWR Morbidity and mortality weekly report. 2014;63(29):620. 
10. Burdette AM, Webb NS, Hill TD, Jokinen-Gordon H. Race-specific trends in HPV vaccinations and provider recommendations: persistent disparities or social progress? Public health. 2017;142:16776.doi: https://doi.org/10.1016/j.puhe.2016.07.009

11. Williams WW. Surveillance of vaccination coverage among adult populations-United States, 2014. MMWR Surveillance Summaries. 2016;65.

12. Mortensen GL, Adam M, Idtaleb L. Parental attitudes towards male human papillomavirus vaccination: a pan-European cross-sectional survey. BMC public health. 2015;15(1):624. doi:10.1186/s12889-015-1863-6

13. Brewer NT, Fazekas KI. Predictors of HPV vaccine acceptability: a theory-informed, systematic review. Preventive medicine. 2007;45(2-3):107-14.doi: https://doi.org/10.1016/j.ypmed.2007.05.013

14. Katz IT, Ware NC, Gray G, Haberer JE, Mellins CA, Bangsberg DR. Scaling up human papillomavirus vaccination: a conceptual framework of vaccine adherence. Sexual health. 2010;7(3):279-86.doi: https://doi.org/10.1071/SH09130

15. Zimet GD. Improving adolescent health: focus on HPV vaccine acceptance. Journal of Adolescent Health. 2005;37(6):S17-S23.doi: https://doi.org/10.1016/j.jadohealth.2005.09.010

16. Zimet GD, Liddon N, Rosenthal SL, Lazcano-Ponce E, Allen B. Psychosocial aspects of vaccine acceptability. Vaccine. 2006;24:S201-S9.doi: https://doi.org/10.1016/j.vaccine.2006.06.017

17. Gottvall M, Larsson M, Höglund AT, Tydén T. High HPV vaccine acceptance despite low awareness among Swedish upper secondary school students. The European Journal of Contraception \& Reproductive Health Care. 2009;14(6):399-405.doi: https://doi.org/10.3109/13625180903229605

18. Dempsey AF, Abraham LM, Dalton V, Ruffin M. Understanding the reasons why mothers do or do not have their adolescent daughters vaccinated against human papillomavirus. Annals of epidemiology. 2009;19(8):531-8.doi: https://doi.org/10.1016/j.annepidem.2009.03.011

19. Lloyd GP, Marlow LA, Waller J, Miles A, Wardle J. An experimental investigation of the emotional and motivational impact of HPV information in adolescents. Journal of Adolescent Health. 2009;45(5):532-4.doi: https://doi.org/10.1016/j.jadohealth.2009.06.003

20. Kahn JA, Rosenthal SL, Jin Y, Huang B, Namakydoust A, Zimet GD. Rates of human papillomavirus vaccination, attitudes about vaccination, and human papillomavirus prevalence in young women. Obstetrics \& Gynecology. 2008;111(5):1103-10.doi: 10.1097/AOG.0b013e31817051fa

21. Hendry M, Lewis R, Clements A, Damery S, Wilkinson C. "HPV? Never heard of it!": a systematic review of girls' and parents' information needs, views and preferences about human papillomavirus vaccination. Vaccine. 2013;31(45):5152-67.doi: https://doi.org/10.1016/j.vaccine.2013.08.091

22. Patel H, Jeve YB, Sherman SM, Moss EL. Knowledge of human papillomavirus and the human papillomavirus vaccine in European adolescents: a systematic review. Sex Transm Infect. 2016;92(6):474-9.doi: http://dx.doi.org/10.1136/sextrans-2015-052341

23. Malakouti J, Mirghafourvand M, Gorbani M, Salehi Poormehr H, Pourasad Shahrak S, Jafari Shabiri M. Incidence of Human Papilloma Virus (HPV) infection and its relevant factors among women 
referring to Alzahra Therapeutic-Educational Center of Tabriz, September 2013 to March 2014. The Iranian Journal of Obstetrics, Gynecology and Infertility. 2016;18(185):16-22.

24. Graneheim UH, Lundman B. Qualitative content analysis in nursing research: concepts, procedures and measures to achieve trustworthiness. Nurse education today. 2004;24(2):105-12.doi: https://doi.org/10.1016/j.nedt.2003.10.001

25. Hosseini SV, Anoosheh M, Abbaszadeh A, Ehsani M. Causal Conditions of Adolescent Girls' Tendency toward Physical Activities. Journal of Qualitative Research in Health Sciences. 2015;3(4):331-9.

26. Schwandt TA, Lincoln YS, Guba EG. Judging interpretations: But is it rigorous? Trustworthiness and authenticity in naturalistic evaluation. New directions for evaluation. 2007;2007(114):11-25.doi: https://doi.org/10.1002/ev.223

27. Lauridsen El, Higginbottom G. The roots and development of constructivist grounded theory. Nurse Researcher. 2014;21(5).doi: 10.7748/nr.21.5.8.e1208

28. Stephens DP, Tamir H, Thomas TL. Factors motivating HPV vaccine uptake among vaccinated and nonvaccinated Hispanic young adult women. Hispanic Health Care International. 2016;14(4):18491.doi: https://doi.org/10.1177/1540415316679808

29. Fontenot HB, Fantasia HC, Zimet GD. Increasing HPV Vaccination and Eliminating Barriers: Recommendations From Young Men Who Have Sex With Men. Journal of Adolescent Health. 2017;60(2):S124-S5.

30. Loke AY, Kwan ML, Wong Y-T, Wong AKY. The uptake of human papillomavirus vaccination and its associated factors among adolescents: a systematic review. Journal of primary care \& community health. 2017;8(4):349-62.doi: https://doi.org/10.1177/2150131917742299

31. Morales-Campos DY, Markham CM, Peskin MF, Fernandez ME. Hispanic mothers' and high school girls' perceptions of cervical cancer, human papilloma virus, and the human papilloma virus vaccine. Journal of Adolescent Health. 2013;52(5):S69-S75.doi:

https://doi.org/10.1016/j.jadohealth.2012.09.020

32. Katz IT, Nkala B, Dietrich J, Wallace M, Bekker L-G, Pollenz K, et al. A qualitative analysis of factors influencing HPV vaccine uptake in Soweto, South Africa among adolescents and their caregivers. PloS one. 2013;8(8):e72094.doi: doi:10.1371/journal.pone.0072094

33. Chan Z, Chan T, Lam Y, Lau L, Li K, Tam W. HPV vaccination in Hong Kong: Implications for medical education. Asian Pac J Cancer Prev. 2011;12(4):1095-9.

34. Holman DM, Benard V, Roland KB, Watson M, Liddon N, Stokley S. Barriers to human papillomavirus vaccination among US adolescents: a systematic review of the literature. JAMA pediatrics. 2014;168(1):76-82.doi: 10.1001/jamapediatrics.2013.2752

35. Kwan TT, Chan KK, Yip AM, Tam K, Cheung AN, Lee P, et al. Barriers and facilitators to human papillomavirus vaccination among Chinese adolescent girls in Hong Kong: a qualitative-quantitative study. Sexually transmitted infections. 2008;84(3):227-32.doi:

http://dx.doi.org/10.1136/sti.2007.029363

Page 15/21 
36. Marlow LA, Wardle J, Waller J. Attitudes to HPV vaccination among ethnic minority mothers in the UK: an exploratory qualitative study. Human Vaccines. 2009;5(2):105-10.doi: https://doi.org/10.4161/hv.5.2.7368

37. Williams K, Forster A, Marlow L, Waller J. Attitudes towards human papillomavirus vaccination: a qualitative study of vaccinated and unvaccinated girls aged 17-18 years. J Fam Plann Reprod Health Care. 2011;37(1):22-5.doi: http://dx.doi.org/10.1136/jfprhc.2010.0017

38. Waller J, Marlow LA, Wardle J. Mothers' attitudes towards preventing cervical cancer through human papillomavirus vaccination: a qualitative study. Cancer Epidemiology and Prevention Biomarkers. 2006;15(7):1257-61.doi: 10.1158/1055-9965.EPI-06-0041

39. Haesebaert J, Lutringer-Magnin D, Kalecinski J, Barone G, Jacquard A-C, Régnier V, et al. French women's knowledge of and attitudes towards cervical cancer prevention and the acceptability of HPV vaccination among those with 14-18 year old daughters: a quantitative-qualitative study. BMC public health. 2012;12(1):1034.doi: https://doi.org/10.1186/1471-2458-12-1034

40. Cover JK, Nghi NQ, LaMontagne DS, Huyen DTT, Hien NT. Acceptance patterns and decision-making for human papillomavirus vaccination among parents in Vietnam: an in-depth qualitative study postvaccination. BMC public health. 2012;12(1):629. doi:https://doi.org/10.1186/1471-2458-12-629

41. Gottvall M, Stenhammar C, Grandahl M. Parents' views of including young boys in the Swedish national school-based HPV vaccination programme: a qualitative study. BMJ open. 2017;7(2):e014255.

42. Francis SA, Battle-Fisher M, Liverpool J, Hipple L, Mosavel M, Soogun S, et al. A qualitative analysis of South African women's knowledge, attitudes, and beliefs about HPV and cervical cancer prevention, vaccine awareness and acceptance, and maternal-child communication about sexual health. Vaccine. 2011;29(47):8760-5.doi: http://dx.doi.org/10.1136/bmjopen-2016-014255

43. Cruz MRID, Tsark JAU, Chen JJ, Albright CL, Braun KL. Human papillomavirus (HPV) vaccination motivators, barriers, and brochure preferences among parents in multicultural Hawai 'i: a qualitative study. Journal of Cancer Education. 2017;32(3):613-21.doi: https://doi.org/10.1007/s13187-0161009-2

44. Perkins RB, Clark JA, Apte G, Vercruysse JL, Sumner JJ, Wall-Haas CL, et al. Missed opportunities for HPV vaccination in adolescent girls: a qualitative study. Pediatrics. 2014;134(3):e666-e74.doi: https://doi.org/10.1542/peds.2014-0442

45. Katz ML, Krieger JL, Roberto AJ. Human papillomavirus (HPV): college male's knowledge, perceived risk, sources of information, vaccine barriers and communication. Journal of men's health. 2011;8(3):175-84.doi: https://doi.org/10.1016/j.jomh.2011.04.002

46. Perkins RB, Tipton H, Shu E, Marquez C, Belizaire M, Porter C, et al. Attitudes toward HPV vaccination among low-income and minority parents of sons: a qualitative analysis. Clinical pediatrics. 2013;52(3):231-40.doi: https://doi.org/10.1177/0009922812473775

47. Al-Dubai S, Alshagga MA, Al-Naggar RA, Al-Jashamy K, Baobaid MF, Tuang CP, et al. Knowledge, attitudes and barriers for human papilloma virus (HPV) vaccines among Malaysian women. Asian 
Pac J Cancer Prev. 2010;11(4):887-92.

48. Fontenot HB, Fantasia HC, Vetters R, Zimet GD. Increasing HPV vaccination and eliminating barriers: Recommendations from young men who have sex with men. Vaccine. 2016;34(50):6209-16.doi: https://doi.org/10.1016/j.vaccine.2016.10.075

49. Ferrer HB, Trotter CL, Hickman M, Audrey S. Barriers and facilitators to uptake of the school-based HPV vaccination programme in an ethnically diverse group of young women. Journal of Public Health. 2015.doi: 10.1093/pubmed/fdv073

50. Yarmohammadi S, Taheri G, Mousavi SS, Sheikhehpour M, Paykoub MH, Hashemian AH. The effect of education on knowledge, attitude and practice of patients with vaginitis. Adv Biol Res. 2015;9(3):196-200.doi: 10.5829/idosi.abr.2015.9.3.9416

51. Batista Ferrer H, Trotter CL, Hickman M, Audrey S. Barriers and facilitators to uptake of the schoolbased HPV vaccination programme in an ethnically diverse group of young women. Journal of Public Health. 2016;38(3):569-77.doi: https://doi.org/10.1093/pubmed/fdv073

52. Tissot AM, Zimet GD, Rosenthal SL, Bernstein DI, Wetzel C, Kahn JA. Effective strategies for HPV vaccine delivery: the views of pediatricians. Journal of Adolescent Health. 2007;41(2):119-25.doi: https://doi.org/10.1016/j.jadohealth.2007.05.007

\section{Tables}

Table 1: Characteristics of young adults in Study of Factors Affecting HPV vaccine uptake (n=10)

\begin{tabular}{|c|c|c|}
\hline Variables & Group & Frequency(\%) \\
\hline \multirow[t]{2}{*}{ Gender } & male & $3(30.0)$ \\
\hline & female & $7(70.0)$ \\
\hline \multirow[t]{2}{*}{ Education } & Diploma and lower & $3(30.0)$ \\
\hline & Academic & $4(70.0)$ \\
\hline \multirow[t]{3}{*}{ Employment status } & Unemployed & $4(40.0)$ \\
\hline & Non-employee & $2(20.0)$ \\
\hline & Employed & $4(40.0)$ \\
\hline \multirow[t]{3}{*}{ Marital status } & Single & $4(40.0)$ \\
\hline & Married & $5(50.0)$ \\
\hline & Divorced & $1(10.0)$ \\
\hline \multirow[t]{2}{*}{ Income } & Low & $4(40.0)$ \\
\hline & Moderate & $6(60.0)$ \\
\hline \multirow[t]{2}{*}{ Age } & Men & Standard deviation \\
\hline & 25.10 & 3.14 \\
\hline
\end{tabular}

Table 2: Characteristics of health professionals in Study of Factors Affecting HPV vaccine uptake $(n=20)$ 


\begin{tabular}{|c|c|c|}
\hline Variables & Group & Frequency(\%) \\
\hline \multirow[t]{2}{*}{ Gender } & male & $7(35)$ \\
\hline & female & 13(65) \\
\hline \multirow[t]{3}{*}{ Education } & Professional doctorate & $10(50)$ \\
\hline & PHD & $9(45)$ \\
\hline & Postgraduate & $1(5)$ \\
\hline \multirow[t]{3}{*}{ Employment status } & Physician & $5(25)$ \\
\hline & Professor & $7(35)$ \\
\hline & Professor and Physician & $8(40)$ \\
\hline \multirow[t]{4}{*}{ Employment experience } & 10 years and above & $6(30)$ \\
\hline & 11 to 20 years & $3(15)$ \\
\hline & 21 to 30 years & $8(40)$ \\
\hline & 31 years and up & $3(15)$ \\
\hline \multirow[t]{3}{*}{ Marital status } & Single & 0 \\
\hline & Married & 18(90) \\
\hline & Divorced & $2(10)$ \\
\hline \multirow[t]{8}{*}{ Specialty type } & psychologist & $4(20)$ \\
\hline & reproductive health & $3(15)$ \\
\hline & urology & $2(10)$ \\
\hline & health education and health promotion & $4(20)$ \\
\hline & dermatology & $2(10)$ \\
\hline & gynecology & $2(10)$ \\
\hline & infectious diseases & $2(10)$ \\
\hline & social practitioner & $1(5)$ \\
\hline \multirow[t]{2}{*}{ Age } & Men & Standard deviation \\
\hline & 1.65 & 0.489 \\
\hline
\end{tabular}

Table 3: Factors affecting uptake and non-uptake of the vaccine among Tehran young adults 


\begin{tabular}{|c|c|}
\hline Category & Sub-category \\
\hline \multirow[t]{2}{*}{ Health value } & Taking care of oneself and others \\
\hline & Responsibility for health \\
\hline \multirow[t]{2}{*}{ Knowledge } & Lack of knowledge about the HPV vaccine, its benefits and HPV disease \\
\hline & Lack of knowledge about health service clinics and the place to purchase the vaccine \\
\hline \multirow[t]{2}{*}{ Outcome expectations } & Fear of the vaccine side effects \\
\hline & Unknown consequences of the HPV vaccine \\
\hline \multirow[t]{2}{*}{ Perceived threat } & Perceived danger \\
\hline & Perceived severity \\
\hline \multirow[t]{2}{*}{ Health services } & Cost \\
\hline & Service coverage \\
\hline \multirow[t]{4}{*}{ Contextual factors } & Education role in the HPV vaccine uptake \\
\hline & sex role in the HPV vac cine uptake \\
\hline & personality characteristics Individuals' \\
\hline & Culture Effects \\
\hline \multirow[t]{5}{*}{ External stimuli } & Peer Effects in the HPV vaccine uptake \\
\hline & Parental Effects in the HPV vaccine uptake \\
\hline & Physicians' Effects in the HPV vaccine uptake \\
\hline & Media Effects in the HPV vaccine uptake \\
\hline & Observational learning \\
\hline
\end{tabular}

Table 4: The strategies for increasing the vaccine uptake in a low-vaccine country 


\section{pulsory vaccination:}

$\mathrm{t}$ of the participants, due to the high cost of the vaccine, demanded that ıealth system, like many other countries, make the HPV vaccine datory and include it in the vaccination schedule. Given the high costs he government, it has been suggested to provide free vaccination for e at risk.
Everyone has to be able to inject the vaccine, and even there should be free vaccination. (psychologist, 31 years old) There should be free vaccination for people at risk. (reproductive health specialist, 55 years old)

\section{sine availability:}

$\mathrm{n}$ the scarcity of the vaccine, the government should provide facilities make it easy for everyone to access the vaccine and make it available fferent groups of society.

\section{sating and informing:}

most important strategy mentioned by the participants was to educate rent groups of people to raise awareness and sexual literacy in the munity. According to the information obtained, the participants anded a public educational process as follows.

\section{dience Groups:}

participants believed that the first step is that the community leaders, as as celebrities, who are influential, including religious people, athletes, rs, should be aware of HPV and its vaccine; since they are as a factor he vaccine uptake.

health system should also be responsible for educating parents, young ts, high-risk individuals, and physicians about the HPV disease and its :ine, as well as training the staff on how to behave with the clients.

\section{lucational Content:}

$\mathrm{n}$ sex education in the country is difficult due to cultural issues and es in the society, the first step in this regard is to educate people about ally transmitted diseases to normalize and desensitization talking about e issues.

community men think because they use condoms as a preventive device, are not at risk of developing sexually transmitted diseases, it is :ssary to educate them about condom error in sexually transmitted ıses.

Educating young parents about sex and sexually transmitted diseases and how to later inform their children was also a part of the participants' suggestions to increase knowledge.
Some vaccine types should be available to uptake the vaccine. (Health Psychologist, 31 years old)
Our artists are influential people. Once, in our religious community, a religious leader had the most power, but now celebrities like athletes, actors and artists have a lot of power (Reproductive Health Specialist, 55 years old)

Even as university professors, we do not have the necessary knowledge in this regard. Knowledge should be given to the health professionals, to the parent, most importantly how parents educate their children (Health Psychologist, 31 years old)

When we talk about this message, the vaccine is important. Injecting this vaccine does not indicate that a person has highrisk relationships. We should normalize and desensitize this issue among the public. (Psychologist, 31 years old)

Educate men about condoms and their error percentage (psychologist, 31 years old)

People can also be a barrier themselves. For the sake of awareness, for example, we want to educate people on how to talk to their youth later (psychologist, 31 years old) 
participants believed that education should take place at all stages of at junior high school and high school levels before sexual activity hermore, education for sexual abstinence when someone is single, ıding What are the high-risk behaviors? Different preventive methods,

ıde pre-marriage counseling on sexually transmitted diseases, including and its related vaccine.

\section{lucational channels:}

participants believed that relevant experts should provide education in ; media, such as television, which plays an essential role in informing le. First sex programs need to be shown on channels, such as health inels and then slowly in popular channels.

эrtising should also be carried out on rural and city health networks, and mation has to be provided to the patients in clinics by physicians.

\section{ow to present the content:}

participants believed that because it is taboo to talk about sex, it is ər to have individual education for young adults.

e are also many models of behavior change in health education. The ; important of them are the Health Belief Model and the Transtheoretical el and other models that can motivate behavior change. It is advisable to !lop materials on sex and related diseases and their prevention based on e models.

e of the participants believed that media education is not valuable; since one-sided, and it is better to have face-to-face and question and answer :ation.

\section{lucational Conditions:}

cation should take place in a way that information is told very slowly consistently, and then acceptance and analysis of the contents should be $\therefore$ It requires planning and compulsion.

arding sensitive issues, such as sex, a supportive environment should be ted based on health promotion principles both in education and the rest e stages.
Sexual abstinence is the practice of refraining from some or all aspects of sexual activity until marriage, educateding what highrisk behavior means, and how to prevent it

They can also include education in premarital consultation (urologist, 46 years old)

Mass media, radio, and television will play an important role in vaccine uptake (dermatologist, 64 years old)

Doctor is very effective in uptake the vaccine. For example, you go for another illness. The doctor then gives you this information, or tells you for example, this is a new disease, and you must do this to prevent it and so on. (young participant, 27 years old)

In my opinion on stigma issues such as HPV disease, individual counseling should be provided (health education specialist, 46 years old)

We discuss how social liberation in the transtheoretical model can affect other people, and also how the incentive control construct can increase the motivation to uptake the vaccine among people. (Health education and health promotion specialist, 36 years old)

The media is one-sided, such as television, and we have no contact with people. However, when someone explains to 20 mothers in person, if one disagrees and talks about the issue, there will be a response. But the TV does not answer (young participant, 25 years old)

Hospitals have taken a number of steps. Educational pamphlets have been provided for patients in educational hospitals. They can play a role if they are planned and mandatory. (Infectious disease specialist, 66 years old)

When we want to inform people about a sensitive issue in society, such as sex issues, the first thing we have to do with health promotion principles is to create a supportive environment. (Reproductive health specialist, 55 years old) 